\title{
Conducting Cytocompatible Scaffolds: Composite of Sodium Hyaluronate and Colloidal Particles of Conducting Polymers
}

\section{Thanh Huong Truong}

Tomas Bata University in Zlin

Lenka Musilová

Tomas Bata University in Zlin

Věra Kašpárková

Tomas Bata University in Zlin

Daniela Jasenská

Tomas Bata University in Zlin

\section{Petr Ponížil}

Tomas Bata University in Zlin

\section{Antonín Minařík}

Tomas Bata University in Zlin

\section{Eva Korábková}

Tomas Bata University in Zlin

\section{Aleš Mráček}

Tomas Bata University in Zlin

\section{Petra Rejmontová}

Tomas Bata University in Zlin

Petr Humpolíček ( $\nabla$ humpolicek@utb.cz)

Tomas Bata University in Zlin

\section{Research Article}

Keywords: Conducting scaffold, Sodium Hyaluronate, Polyaniline, Polypyrrole, Cytocompatibility

Posted Date: November 30th, 2021

DOI: https://doi.org/10.21203/rs.3.rs-1101105/v1

License: (1) (1) This work is licensed under a Creative Commons Attribution 4.0 International License.

Read Full License 


\section{Abstract}

Novel bio-inspired conductive scaffolds composed of sodium hyaluronate containing water soluble polyaniline or polypyrrole colloidal particles (concentrations $0.108,0.054$ and $0.036 \% \mathrm{w} / \mathrm{w}$ ) were manufactured. For this purpose, either crosslinking with $\mathrm{N}$-(3-dimethylaminopropyl- $\mathrm{N}$-ethylcarbodiimide hydrochloride and $\mathrm{N}$-hydroxysuccinimid or a freeze-thawing process in the presence of poly(vinylalcohol) were used. The scaffolds comprised interconnected pores with prevailing porosity values of $\sim 30 \%$ and pore sizes enabling the accommodation of cells. Good swelling capacity (92-97\%) without any sign of disintegration was typical for all samples. The elasticity modulus depended on the composition of the scaffolds, with the highest value of $\sim 50000 \mathrm{~Pa}$ obtained for the sample containing the highest content of polypyrrole particles. The scaffolds did not possess cytotoxicity and allowed cell adhesion and growth on the surface. Using the in vivo-mimicking conditions in a bioreactor, cells were also able to grow into the structure of the scaffolds. The technique of scaffold preparation used here thus overcomes the limitations of conducting polymers (e.g. poor solubility in an aqueous environment, and limited miscibility with other hydrophilic polymer matrices) and moreover leads to the preparation of cytocompatible scaffolds with potentially cell-instructive properties, which may be of advantage in the healing of damaged electro-sensitive tissues.

\section{Introduction}

Bioelectrical signals are one of the key players in the development and regeneration of tissues ${ }^{1}$. Due to this fact, electrical stimulation has been studied as a promising tool in disease treatment and wound healing 2 for decades. In this context, the development of novel stimuli-responsive electroconductive biomaterials is of great interest when considering the use of such materials in a wide variety of biomedical applications ${ }^{3}$. Indeed, to facilitate electrical stimulation, it is crucial to use electrically conductive biomaterials. Here, different class of materials can be considered; these include metals, conductive polymers, and piezoelectric materials, which can all transfer electrical, electrochemical, and potentially even electromechanical stimuli to cells ${ }^{4}$. Recently, the preparation of electrically conductive polymers (CP), such as polypyrrole (PPy) and polyaniline (PANI), has become a highly prized goal of the tissue engineering community, as they can efficiently transfer electrical signals at the interfaces with cells due to their intrinsically combined electronic and ionic conductivity ${ }^{5}$. Owing to ionic conductivity, the electrical signal can be transduced to an ionic signal, which is a natural communication system of cells ${ }^{6}$. Consequently, CPs can be highly beneficial in tissue engineering (TE), especially of cardiac, muscle, and nerve tissues ${ }^{7,8}$. Among additional advantages of CPs are their easy synthesis. Both PANI and PPy can be prepared in various forms such as powders ${ }^{9}$, colloidal dispersions ${ }^{10}$ or films ${ }^{11}$, of which the form facilitating the processing and/or preparation of composites (colloidal dispersion) is especially important. Another shortcoming of CPs is their inability to be prepared in a three-dimensional form (scaffolds). Therefore, naturally derived polymers, such as sodium hyaluronate (HA), have been employed to overcome the limitations related to the fabrication of suitable conducting 3D scaffolds with required mechanical, viscoelastic, and biological properties ${ }^{12}$. Recently, many studies have demonstrated that HA 
plays a crucial role in various biological processes, including cell proliferation, migration, and differentiation, or in the activation of anti-inflammatory growth factors ${ }^{13,14}$. Sodium hyaluronate is a naturally-occurring glycosaminoglycan exhibiting intriguing viscoelastic properties, excellent biocompatibility, biodegradability, and the ability to be easily modified with various functional groups ${ }^{15}$. Moreover, it possesses other benefits for TE, including the absence of toxicity and non-thrombogenic and nonimmunogenic properties, in contrast to other synthetic materials ${ }^{16}$. As the constituent of the extracellular matrix, $\mathrm{HA}$ is a candidate component for use in the synthesis of scaffolding materials for TE. Collier et al. demonstrated that HA-doped PPy improved surface morphology and biocompatibility, and significantly promoted vascularization in vivo in comparison with poly(styrenesulfonate)-doped PPy ${ }^{17}$. The molecular weight of the polymer also plays an important role in the hydrophilicity or cell receptor signalling of HAbased materials ${ }^{18}$. Kim et al. reported that the behaviour of PPyHA based biomaterials differed according to the molecular weight (MW) of $\mathrm{HA}$ (in the range $35 \times 10^{3}-2 \times 10^{6} \mathrm{Da}$ ), with PPy/high-MW HA exhibiting higher electrochemical activity, lower impedance, and higher hydrophilicity. Meanwhile, PPy/lower-MW HA supported the adhesion and growth of fibroblasts and neuronal cells, highMW HA-doped PPy resisted cell growth due to the higher hydrophilicity of its surface ${ }^{19}$. According to Shin et al., HA hydrogel significantly promoted the neuronal differentiation of human fetal neural stem cells and human-induced pluripotent stem cell-derived neural progenitor cells ${ }^{20}$. Texidó et al. reported the modification of PPy with dopaminated hyaluronic acid, which improved adhesion onto a poly(dimethylsiloxane) substrate, thus forming a flexible composite film. The film demonstrated enhanced water resistance and electrical conductivity under mechanical stimuli compared with bare PPy films and PPy with undopaminated hyaluronic acid ${ }^{21}$. So far only a limited number of studies have been reported dealing with the use of PANI and HA for the fabrication of electrically conductive scaffolds. One recent study dealing with this topic highlighted the induction of cardiomyocyte proliferation within lamellar scaffolds based on a colloidal complex of water-soluble PANI with collagen, fibroin, and hyaluronate 22 .

However, as far as the authors know, no previously published study has investigated the scaffolds presented here, these prepared by the chemical and physical crosslinking of sodium hyaluronate (HA) with incorporated CP-based colloidal particles. In this work, such scaffolds were prepared using embedded conducting and water soluble colloidal PPy or PANI particles stabilized also with HA. The main aim of the study was to investigate whether - and if so, how - the method of crosslinking and the composition of the material influence the physico-chemical and biological properties of the scaffolds in terms of cytocompatibility and the ability to facilitate the ingrowth of mouse embryonic fibroblast cells. This novel and original approach was investigated with the aim of preparing porous, conductive, and cytocompatible scaffolds for TE.

\section{Materials And Methods}

Materials 
Reagent-grade aniline hydrochloride ( $\geq 98 \%$ ), pyrrole (98\%), ammonium peroxydisulfate (98\%) and poly(vinyl alcohol) (PVA; molecular weight $40 \cdot 10^{3} \mathrm{~g} \cdot \mathrm{mol}^{-1}$ ) were purchased from Sigma Aldrich (Germany) as well as the crosslinking agents, $N$-(3-dimethylaminopropyl- $N$-ethylcarbodiimide hydrochloride (EDC) and N-hydroxysuccinimid (NHS). Sodium hyaluronate (HA; molecular weight 1.8-2.1 $\cdot 10^{6} \mathrm{~g} \cdot \mathrm{mol}^{-1}$ ) was a kind gift from Contipro a.s. (Czech Republic).

Synthesis of polyaniline and polypyrrole colloids

Samples of colloidal PANI and PPy were prepared via the oxidation of the respective monomer, aniline hydrochloride (AH) or pyrrole (Py), with ammonium peroxydisulfate (APS) in the presence of sodium hyaluronate (HA). The colloidal particles are designated HaPANI and HaPPy. Briefly, HA was dissolved to a $1 \%(\mathrm{w} / \mathrm{w})$ solution in MilliQ water under stirring at $55^{\circ} \mathrm{C}$ overnight. For dispersion polymerization, $\mathrm{AH}$ $(0.2 \mathrm{M})$ in $1 \%(\mathrm{w} / \mathrm{w}) \mathrm{HA}$ solution was prepared, and polymerization was started at room temperature by adding $0.1 \mathrm{M}$ aqueous APS solution to the reaction mixture. The mixture was stirred for $5 \mathrm{~min}$, and left at rest to polymerize. The reaction was completed within $2 \mathrm{~h}$. Correspondingly, PPy was prepared using $0.2 \mathrm{M}$ Py monomer solution in $1 \%(\mathrm{w} / \mathrm{w}) \mathrm{HA}$ and $0.25 \mathrm{M}$ aqueous APS, which were mixed and left to polymerize for $2 \mathrm{~h}$. In order to remove residual impurities, the colloidal dispersions were transferred into Spectra Por 2 membrane dialysis tubing (cut-off 12000 - 14 000; Spectrum Laboratories Inc., U.S.) and purified through exhaustive dialysis against $0.2 \mathrm{M}$ hydrochloric acid. Each of the colloidal dispersions was employed for the preparation of scaffolds.

Preparation of scaffolds

Samples were prepared in two different ways, specifically by means of chemical $\left.{ }^{(\mathrm{Ch}}\right)$ and physical $\left({ }^{\mathrm{Ph}}\right)$ crosslinking procedures. The composition of the samples is given in Table 1.

On the basis of preliminary studies, the hyaluronan scaffolds containing PANI or PPy colloids chemically crosslinked with EDC and NHS (designated $\mathrm{HaPANI}{ }^{\mathrm{Ch}}, \mathrm{HaPPy}{ }^{\mathrm{Ch}}$ ) were made according to ${ }^{23}$. A stock solution of $\mathrm{HA}$ with a concentration of $1 \%(\mathrm{w} / \mathrm{w})$ was prepared by dissolving the polymer in Milli-Q water for $24 \mathrm{~h}$ at $50^{\circ} \mathrm{C}$. The crosslinking agents EDC $(100 \mathrm{mM})$ and NHS were added to the $1 \%(\mathrm{w} / \mathrm{w}) \mathrm{HA}$ solution in a ratio of $1: 1.5(\mathrm{w} / \mathrm{w})$ and the mixture was stirred for $1 \mathrm{~h}$ at $25^{\circ} \mathrm{C}$. Subsequently, the solution was titrated under continuous stirring, with PANI colloid dispersed in $0.2 \mathrm{M} \mathrm{HCl}$ to adjust the $\mathrm{pH}$ to $4.5-$ 4.7. Correspondingly, PPy-containing scaffolds were prepared.

The crosslinking of scaffolds using the physical freeze-thaw method ${ }^{24-26}$ was carried out using PVA, and the samples were designated $\mathrm{HaPANI}^{\mathrm{Ph}}, \mathrm{HaPPy}{ }^{\mathrm{Ph}}$. A stock solution of PVA with a concentration of $10 \%(\mathrm{w} / \mathrm{w})$ was prepared by dissolving the polymer in Milli-Q water for $24 \mathrm{~h}$ at $80^{\circ} \mathrm{C}$. The PVA was added to the $1 \%(\mathrm{w} / \mathrm{w}) \mathrm{HA}$ solution in a ratio $1: 2(\mathrm{w} / \mathrm{w})$ and stirred for $1 \mathrm{~h}$ at $25^{\circ} \mathrm{C}$. Subsequently, the solution was titrated under continuous stirring, with the colloidal dispersion of PANI (in $0.2 \mathrm{M} \mathrm{HCl}$ ) to adjust the pH to 4.5-4.7. Correspondingly, PPy containing scaffolds were prepared. The concentrations of CP-based 
colloidal particles in the scaffold were $0.108,0.054$ and $0.036 \%(\mathrm{w} / \mathrm{w})$, these values used as superscripts for sample labelling.

The solutions from both preparation routes (chemical or physical) were then poured into polystyrene moulds and first frozen at $-18^{\circ} \mathrm{C}$ for $60 \mathrm{~h}$, then freeze-dried in an ALPHA1-2 LD plus freeze-dryer (Christ, UK). As a result, cylindrical specimens were obtained with a diameter of $28 \mathrm{~mm}$ and a height of $5 \mathrm{~mm}$.

Table 1

Composition of scaffolds. The samples are labelled according to the type of CP-based colloidal particles (PANI, PPy); the method of crosslinking, i.e. chemical (Ch) or physical (Ph); and the concentration of CP-based colloidal particles.

\begin{tabular}{|c|c|c|c|c|c|c|}
\hline \multirow[t]{2}{*}{ Sample } & \multicolumn{6}{|c|}{ Composition (w/w) } \\
\hline & HA & PVA & PANI & PPy & NHS & EDC \\
\hline HaPANICh_108 & 1 & $x$ & 0.108 & $x$ & 0.013 & 0.013 \\
\hline HaPANICh_54 & 1 & $x$ & 0.054 & $x$ & 0.013 & 0.013 \\
\hline HaPANICh_36 & 1 & $x$ & 0.036 & $x$ & 0.013 & 0.013 \\
\hline HaPPyCh_108 & 1 & $x$ & $x$ & 0.108 & 0.013 & 0.013 \\
\hline HaPPy & 1 & $x$ & $x$ & 0.054 & 0.013 & 0.013 \\
\hline HaPPyCh_36 & 1 & $x$ & $x$ & 0.036 & 0.013 & 0.013 \\
\hline HaPANI ${ }^{\text {Ph_108 }}$ & 1 & 0.5 & 0.108 & $x$ & $x$ & $x$ \\
\hline HaPANI ${ }^{\text {Ph_54 }}$ & 1 & 0.5 & 0.054 & $x$ & $x$ & $x$ \\
\hline HaPANI ${ }^{\text {Ph_36 }}$ & 1 & 0.5 & 0.036 & $x$ & $x$ & $x$ \\
\hline HaPPyPh_108 & 1 & 0.5 & $x$ & 0.108 & $x$ & $x$ \\
\hline HaPPyPh_54 & 1 & 0.5 & $x$ & 0.054 & $x$ & $x$ \\
\hline HaPPyPh_36 & 1 & 0.5 & $x$ & 0.036 & $x$ & $x$ \\
\hline
\end{tabular}

Physico-chemical characterization

Size and morphology of colloidal particles

The particle size and size distribution were determined on dialysed samples using dynamic light scattering (DLS) (Zetasizer Nano ZS, Malvern Instrument, UK). The hydrodynamic radii of colloidal particles, expressed as z-average particle diameters, were measured at $25^{\circ} \mathrm{C}$ at a scattering angle of $173^{\circ}$. The polydispersity index (PDI) describing the width of the particle size distribution in a given sample was 
also determined. Samples were prepared for measurement by diluting $10 \mu \mathrm{L}$ of freshly prepared dispersion in $1 \mathrm{~mL}$ of $0.1 \mathrm{M} \mathrm{HCl}$. The analyses were run in triplicates with average values and standard deviations reported.

The morphology of colloidal particles was assessed with a transmission electron microscope (TEM) JEOL JEM 2000 FX (Japan).

\section{Mechanical properties}

Mechanical properties in terms of Young's modulus were determined on an Autograph AG-X tensile tester (Shimadzu). The analyses were performed on cylindrical samples with a length of $12 \mathrm{~mm}$ and a diameter of $8 \mathrm{~mm}$. Each sample was inserted between two horizontal plates and deformed at a rate of $1 \mathrm{~mm}$ $\min ^{-1}$. The measurement time was $3 \mathrm{~min}$ and within this short period of time the humidity of the sample was considered constant. Young's modulus was determined as the slope of the linear part of the stressstrain curve. The experiments were performed in compression mode and the relative standard deviation of all values obtained did not exceed $5 \%$.

\section{Surface topography and electrical properties}

The surface electrical properties of the scaffolds were analysed by tunneling atomic force microscopy (AFM) using the PeakForce TUNA module on a Dimension ICON instrument (Bruker Corporation; US). Measurements were performed at ambient temperature using a PFTUNA probe (Bruker Corporation; US) with a spring constant of $0.4 \mathrm{~N} \mathrm{~m}^{-1}$, covered on both sides with a conducting Pt/lr layer. The scanning rate was $0.3 \mathrm{~Hz}$ and the TUNA and peak currents were measured by applying a bias voltage of $10 \mathrm{~V}$. The resulting parameters of area roughness and TUNA and peak currents were determined according to the protocol of ISO 25178-2 standard using NanoScope Analysis software v. 1.5. The following parameters were determined: surface roughness $\left(S_{a}\right)$, maximal surface height $\left(S_{z}\right)$, average and maximum TUNA current, and average and maximum peak current. The TUNA current is the average current over one full tapping cycle in PeakForce mode. Peak current corresponds to the current measured at a defined maximum force in the PeakForce cycle. Data from AFM were processed using Gwyddion 2.5 software (Czech Metrology Institute, Czech Republic).

\section{Morphology and porosity of scaffolds}

The surface morphology of lyophilized samples was studied using a scanning electron microscope (Phenom Pro desktop scanning electron microscope (SEM) with a BSE detector (Phenom-World B.V., The Netherlands)) at acceleration voltages of 10 and $15 \mathrm{kV}$. The samples were cut into sections using a sharp razor blade, to observe the cross-sectional morphologies of their scaffolds. The pore sizes of the scaffolds were measured by analysing the SEM images captured as described above. The method of image analysis was based on the chord length method used in the ASTM standard ASTM E-112 (E-112, 2010). A system of intersecting lines was drawn on the SEM images of the planar sections of the samples, and chord lengths were automatically measured by an in-house algorithm written in the Python 
programming language. Then, the mean pore size was computed using the protocol of ASTM E-112 standard and expressed as a percentage of pore volume relative to the total volume of the sample.

\section{Swelling of scaffolds}

The swelling degree $(S D)$ of the lyophilized samples was determined gravimetrically. Weighted, diskshaped specimens were immersed in phosphate buffered saline (PBS) at $37^{\circ} \mathrm{C}$ to reach swelling equilibrium. The water uptake was determined by removing the swelled samples from the PBS at selected time intervals, wiping them with tissue paper, and weighing them. The $S D$ was calculated according to the equation $S_{D}=\left(W_{S}-W_{D}\right) / W_{D} \cdot 100(\%)$, where $W_{S}$ and $W_{D}$ are the weights of the swollen and dry sample, respectively. The presented results are average values from four independent measurements.

Cytocompatibility of scaffolds

The cytocompatibility of the scaffolds was determined using the ATCC CRL-1658 NIH/3T3 mouse embryonic fibroblast cell line. ATCC-formulated Dulbecco's Modified Eagle's Medium, catalogue no. 302002, containing $10 \%$ calf serum (BioSera, France) and penicillin/streptomycin, $100 \mathrm{U} \mathrm{mL}^{-1}$ (GE Healthcare HyClone, UK) was used as the cultivation medium in all experiments.

\section{Cytotoxicity}

The cytotoxicity was investigated according to ISO protocol 10993-5, both on freshly prepared scaffolds and after their lyophilization. For the test, $0.1 \mathrm{~g}$ of tested material was extracted with $1 \mathrm{~mL}$ of cultivation medium for $24 \mathrm{~h}$ at $37^{\circ} \mathrm{C}$ under stirring. The cells were pre-cultivated for $24 \mathrm{~h}$ and seeded at a concentration of $1 \times 10^{5}$ per well ( 96 well plates were used). The extracts were diluted with culture medium to obtain a series of dilutions with concentrations of $75,50,25,20,10$, and $1 \%$. After incubation for $24 \mathrm{~h}$, the cell viability was determined by 3-(4,5-dimethylthiazol-2-yl)-diphenyltetrazolium assay (MTT cell proliferation assay kit, Duchefa Biochemie, Netherlands). The absorbance was measured at a wavelength of $570 \mathrm{~nm}$ using an Infinite m200pro luminometer (Tecan, Switzerland). The viability of the cells was expressed as the reduction in cell viability relative to the reference, i.e., cells cultivated in the absence of extracts. All tests were conducted in quadruplicates. The morphology of the cells was observed using an inverted Olympus phase contrast microscope (Olympus IX81, Japan).

\section{Proliferation test}

The ability of cells to proliferate on the tested samples after their lyophilisation was determined using the ATCC CRL-1658 NIH/3T3 mouse embryonic fibroblast cell line. A cell suspension at a concentration of 1 $\times 10^{6}$ cells per $\mathrm{mL}$ was prepared, and $0.3 \mathrm{~mL}$ of the suspension was carefully injected by syringe into each scaffold and onto its surface, and placed into an incubator for $2 \mathrm{~h}$ to induce cell adhesion and initiate proliferation. After this period the scaffold was spill over by cultivation medium andcultivated for 7 days. The medium was changed every second day of the experiment. After the cultivation period, the cells were fixed and stained with Hoechst 33258 (Molecular Probes, Carlsbad, CA) and ActinRed 555 (Life 
Technologies, USA). The morphology of the cells was investigated using a confocal microscope (Olympus Fluoview FV 3000, Japan).

\section{Cell ingrowth into scaffolds}

Before testing the scaffolds in the 3D environment of a bioreactor, cells were seeded onto the scaffolds and pre-cultivated for $24 \mathrm{~h}$ under the standard conditions given above. After pre-cultivation, the samples were placed into a bioreactor, where they were cultivated for the next 7 days to allow cell ingrowth. For testing, an RCCS-4 rotary cell culture system (Synthecon Incorporated, USA) was used. Each of the samples was separately inserted inside the bioreactor and $50 \mathrm{~mL}$ of cultivation medium was added. The forward rotation of the reactor was adjusted to $10.5 \mathrm{rpm}$. The partial oxygen pressure, as well as the temperature were the same as under standard cultivation in an incubator. After cultivation, the scaffolds were fixed with $4 \%$ paraformaldehyde overnight, washed with PBS, permeabilized with $0.5 \%$ Triton X-100 (Sigma Aldrich, Germany), and washed again three times with PBS. The cells were stained using ActinRed $^{\text {TM }} 555$ (Thermo Fisher Scientific, USA). The tested samples were sliced, and the cell morphology was observed using a confocal microscope (Olympus Fluoview FV 3000, Japan).

\section{Results And Discussion}

Conducting colloidal dispersions

Colloidal dispersions of CP stabilized with polysaccharides are smart formulations helping to solve the problem of the poor solubility of CP in aqueous environment, and improve the cytocompatibility of the final composite ${ }^{27}$. Another, not previously mentioned advantage of such composites appears in the case when CPs are mixed with other hydrophilic polymer matrices - for example, when polymer-based scaffolds are fabricated. Here, the better polymer-polymer compatibility of HaPANI or HaPPy colloids containing HA combined with the bulk polymer of the scaffold (also based on HA) is expected in comparison with pristine PANI or PPy.

The size and polydispersity index of colloidal particles used for scaffold fabrication were determined on thoroughly dialysed samples, purified of possible impurities which might cause cytotoxic effects ${ }^{28}$. The hydrodynamic diameters of particles were different, with micrometer sizes observed for HaPANI (2200 \pm $260 \mathrm{~nm})$ and smaller sizes determined for HaPPy $(447 \pm 8 \mathrm{~nm})$. Both samples showed, however, similar polydispersity indexes $(\sim 0.2)$. The morphology of particles observed by transmission electron microscopy (Figure 1A and 1B) conformed with scattering measurements (Figure 1C and 1D), showing in both cases regular, spherical particles with a smooth surface, and, in the case of HaPANI, the interconnection of such particles to form bigger, loose clusters.

Preparation and characterization of scaffolds

The CP-based colloidal dispersions HaPANI and HaPPy were used, each incorporated into the used HA matrix and in three different concentrations. Two methods of scaffold preparation were employed, each 
followed by an identical freeze-drying procedure. The first route included chemical crosslinking with EDC and NHS, the latter used as the activator of carboxylic groups; the second procedure involved crosslinking by the freeze-thaw method using PVA, hereinafter referred to as the physical route. The question, hence, arose whether - and if so, how - these two preparation methods together with scaffold composition affect the material and biological properties of the given scaffolds.

Mechanical properties

Mechanical properties defined in terms of the modulus of elasticity $(E)$ were significantly different for samples of HaPPyPh crosslinked by freeze-thawing in the presence of PVA when compared with chemically crosslinked HaPPy ${ }^{\mathrm{Ch}}$ and scaffolds containing HaPANI colloidal particles (Figure 2). Their $E$ values were the highest $(17000-50000 \mathrm{~Pa}$ ) and strongly depended on the content of PPy particles in the samples, with the highest value observed for HaPPyPh_108. A corresponding dependence of the elasticity modulus on the content of colloidal particles was seen for chemically crosslinked HaPPyCh, though with significantly lower $E$ of only $5000-17000 \mathrm{~Pa}$. This indicates a stronger network for the samples containing PVA and good mutual compatibility between all the involved components, HA, PVA, and CP-based colloidal particles, of which the last component contributed to the good level of compatibility through the acidic nature of the particle dispersion ${ }^{29}$. Differences between physically and chemically crosslinked samples containing HaPANI (Figure 2) were only minor in comparison with the corresponding scaffolds prepared with HaPPy. The modulus for physically crosslinked HaPANI ${ }^{\mathrm{Ph}}$ ranged from 5500 to $15000 \mathrm{~Pa}$, with the lowest value observed for the sample with $0.054 \%$ (w/w) colloid (HaPANI ${ }^{\mathrm{Ph}}{ }^{54}$ ). This $E$ range is actually similar to the $E$ range observed for chemically crosslinked samples (HaPANI $\left.{ }^{\mathrm{Ch}}\right)$, i.e., from 5000 to $15000 \mathrm{~Pa}$; however, the correlation between the $E$ values and the amount of colloidal particles is different in these samples. In summary, the scaffolds incorporating HaPANI colloidal particles exhibited a low elasticity modulus, which moreover lacked correlation with the colloid content in the sample. In contrast, the behaviour of all scaffolds containing HaPPy colloid was more predictable in terms of $E v s$ particle content. This is surprising, as one would expect more similar behaviour among scaffolds containing PVA irrespective of the type of colloidal particles used, as PVA contribute to a rubbery and elastic texture and higher mechanical strength of scaffolds thanks to the better distribution of the mechanical load along the crystallites of the three-dimensional scaffold structure ${ }^{30}$. The mechanical properties of the scaffolds hence result from the crosslinking routes and the content/type of colloidal particles used for their fabrication.

Surface topography and electrical properties

Lyophilized samples with a medium concentration of the conducting component $(0.054 \% \mathrm{w} / \mathrm{w})$ were analysed using AFM to determine their surface properties and surface conductivity (Figure 3 ).

The surface roughness $(S a)$ and maximum surface height $(S z)$ of the samples as presented in figure 3 shows that the surface characteristics of the scaffolds are rather similar. Their surfaces are irregular with embedded pores, these seen mainly in physically crosslinked samples. Chemical crosslinking gave rise to 
scaffolds with a flake-like surface structure. The second and third columns of Figure 3 show current maps expressed as TUNA and Peak currents. These unambiguously prove that the scaffolds are conductive and that their average values of TUNA current are similar, lying in the range of $2.1-3.8 \mathrm{pA}$, irrespective of the type of conducting particles used and of the fact that the percentage of the conducting component in the chemically crosslinked samples was higher with respect to the total mass of the scaffold than in the scaffolds crosslinked physically. A bigger difference in the measured values can be seen for maximum currents (max TUNA, max Peak current), which were lower for physically crosslinked samples, with a maximum current of about $12 \mathrm{pA}$. In the case of samples crosslinked chemically, values of 15 and 19 pA for HaPANI ${ }^{C h}{ }^{54}$ and HaPPyCh ${ }^{54}$, respectively, were measured. This minor difference is a natural consequence of the higher ratio of CP-based colloids in the chemically crosslinked samples. In this respect, both HaPANI and HaPPy colloids performed, within the same type of crosslinking mechanism, similarly. Obviously, as the samples contain particles of intrinsically conducting polymer (CP) with mixed ionic and electron conductivity, and as the surroundings within hydrated scaffolds are also ionically conductive, the scaffolds thus form a suitable environment which can facilitate the adhesion and proliferation of electrically conductive cells and tissues ${ }^{20}$.

Porosity and morphology

Morphology, including the size of pores in lyophilized scaffolds, was assessed by means of the visual observation of SEM images (Figure 4). The images were used for the measurements of pore size and the calculation of porosity, which were both quantified according to the chord length method given by the protocol of ASTM E-112 (Figure 5).

According to the results of the image analysis, the physically crosslinked $\mathrm{HaPPy} \mathrm{Ph}^{\mathrm{s}}$ samples with the two lower concentrations of colloidal particles $(0.036,0.054 \%)$ exhibited the biggest pores in the scaffolds. The pore sizes were the smallest in the formulation in which the colloid concentration was $0.108 \%$ (HaPPyPh_108).

In numerical values, the porosity ranged from $26-37 \%$, with the lowest value for the highest content of colloidal particles, as observed for HaPPy ${ }^{\mathrm{Ph}} 108$. The HaPPyCh samples, prepared with chemical crosslinking, yielded a lower porosity of 22 to $28 \%$, which was lowest for the scaffold with the medium content of colloidal particles (HaPPy ${ }^{\mathrm{Ch}}{ }^{54}$ ). The physically crosslinked HaPANI ${ }^{\mathrm{Ph}}$ samples were, with respect to pore sizes, similar to the above-discussed samples containing PPy colloidal particles, and showed pore sizes of 271,288 , and $177 \mu \mathrm{m}$ for scaffolds with increasing concentrations of CP-based colloid. Calculated porosity values for these formulations ranged between 27 and $32 \%$. HaPANICh scaffolds, prepared by chemical crosslinking, exhibited porosity values comparable to those of scaffolds

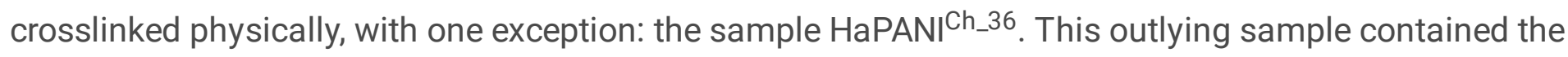
lowest concentration of HaPANI colloidal particles and exhibited a porosity of more than $50 \%$ and an average pore size of $547 \pm 126 \mu \mathrm{m}$. The porosities of the other two HaPANI-based samples were $30 \%$ and $35 \%$ for the medium and highest colloid contents, respectively. According to the image analysis 
supported by visual evaluation, the bigger pores were always seen in samples with the lowest content of colloidal particles and, in general, the sizes of pores in most of the scaffolds were roughly estimated to be within the range of $200-300 \mu \mathrm{m}$, which is sufficient for the ingrowth of cells into the structure and for nutrient supply and metabolite removal.

\section{Swelling}

The course of swelling was similar for all samples, and the initial increase in swelling degree (SD), observed within the first approx. $100 \mathrm{~min}$, was followed by equilibrium (Figure 6). At equilibrium, the $S D$ of physically crosslinked HaPPy ${ }^{\mathrm{Ph}}$ scaffolds ranged from 92 to $96 \%$ and decreased with growing colloid content in the sample. In comparison, differences in the swelling of all chemically crosslinked $\mathrm{HaPPyCh}$ samples were only minor and their equilibrium SD was $93-95 \%$. Scaffolds containing HaPANI exhibited lower $S D$ for physically crosslinked samples in comparison with samples that were crosslinked chemically. With short swelling times, the lowest $S D$ of $87 \%$ was recorded for HaPANI ${ }^{\mathrm{Ph}} 54$, while the highest equilibrium $S D$ of $93 \%$ was observed for the sample containing the highest concentration of particles. Chemically crosslinked HaPANICh scaffolds swelled better, their $S D$ ranging from 95 to $97 \%$. In this respect, $S D$ is influenced by PVA in the hydrogel matrix; in chemically crosslinked samples with PVA absent, the naturally high ability of HA to take up water prevailed and the $S D$ was, therefore, higher. When in a mixture, the crystallinity of PVA affects the hydrogel structure, which is less loose compared with samples containing solely HA. As a result, physically crosslinked gels absorb less water, this resulting in lower $S D$. Nevertheless, regardless of the observed differences, the SD values were similar and all scaffolds showed good swelling characteristics.

\section{Cytotoxicity}

The absence of cytotoxicity is one of the fundamental requirements that biomaterials must meet. The cytotoxicity of a full set of samples was therefore tested. To show representative information, the protocol of ISO 10993-5 standard was employed, including the use of an appropriate cell line; i.e., $\mathrm{NIH} / 3 \mathrm{~T} 3$ fibroblasts. The cytotoxicity was determined on native samples prior to lyophilisation and on lyophilised scaffolds, which are marked with the superscript ${ }^{L}$.

The summary of cytotoxicity data recorded for chemically crosslinked scaffolds and presented in Figure 7 unambiguously demonstrates that all the native scaffolds prepared by chemical crosslinking, $\mathrm{HaPANI}{ }^{\mathrm{Ch}}$ or $\mathrm{HaPPy}$, can be classified as non-cytotoxic, with cell viabilities higher than $70 \%$. The situation was, however different for lyophilised scaffolds. After lyophilisation, the cytotoxicity increased, especially in the case of HaPPy containing scaffolds, the lyophilised HaPPy $\mathrm{Ch}_{-} \mathrm{L}$ scaffolds showing cytotoxic effects with concentrations of extract higher than $25 \%$, irrespective of the concentration of the colloidal particles used. In the case of $\mathrm{HaPANI}^{\mathrm{Ch}}{ }^{\mathrm{L}}$ containing HaPANI colloid, the cytotoxicity was lower and only the two highest concentrations of extracts (70 and 100\%) exceeded the threshold for cytotoxicity, and moreover only slightly. The effect of lyophilisation on cytotoxicity can be related to the protocol of testing given by the ISO standard. The procedure defines the ratio of material/extraction medium according to the mass. 
In the case of lyophilised samples with water removed, the ratio of the mass of dry matter (which in fact contains impurities) to the amount of extraction medium is higher. This situation can lead to the higher cytotoxicity of lyophilized samples, despite the fact that the procedure itself should not produce additional cytotoxic compounds.

The behaviour of physically crosslinked samples (Figure 8) prior to lyophilisation was similar to that observed for scaffolds crosslinked chemically, and only in a few cases did the samples show a cytotoxic effect, which was, moreover, observed at only the highest extract concentration. Only the highest extract concentration of native $\mathrm{HaPANI}^{\mathrm{Ph}}$ scaffold significantly exceeded the cytotoxicity threshold, and the effect correlated with the concentration of HaPANI colloidal particles in the sample. Contrary to the situation observed for scaffolds prepared by chemical crosslinking, here lyophilisation decreased the negative impact of the scaffolds on cell viability. This could actually be observed in the case of all samples, especially in the case of HaPANII ${ }^{\mathrm{Ph} L}$, where none of the lyophilised hydrogel samples was cytotoxic.

The difference in the behaviours of lyophilized scaffolds prepared by chemical and physical crosslinking could, to some extent, be expected, as physically crosslinked scaffolds lack the possible residues of cytotoxic crosslinking agents, which can be released during the lyophlization process. We can also hypothesise that the difference is related to the different pore structures and the type of bound cytotoxic residual compounds within the scaffold structure. It seems that the CP-based colloids presented here do not substantially contribute to the overall cytotoxicity of the scaffolds, either in the case of HaPANI or HaPPy colloidal particles. Indeed, this is not surprising, as previous studies by Humpolicek et al have shown that the cytotoxicities of both CPs are similar ${ }^{31}$.

Cell adhesion, growth and ingrowth

The cytotoxicity of scaffolds, which can be related to the composition of the material and the possible leaching of residual precursors or reagents under extraction was low. The lyophilized samples were therefore subjected to another cytocompatibility study, this involving the determination of bio-interface properties represented by the ability of cells to adhere onto the scaffold surface and subsequently grow. The lyophilized samples were chosen, as their internal architecture was more appropriate for cell ingrowth than that of native scaffolds. The cells were seeded on the surfaces of scaffolds and cultivated for one week. After this time elapsed, the cells were able to adhere and grow on all surfaces. Representative pictures are presented in Figure 9.

Scaffolds suitable for tissue engineering must not only allow the attachment and growth of cells on their surfaces, but also be able to facilitate cell ingrowth into the structure of the scaffold. As mentioned above, an increasing concentration of HaPANI or HaPPy colloidal particles within the scaffold resulted in the higher cytotoxicity of the scaffold. The ingrowth of cells, allowed by their cultivation in a bioreactor mimicking in vivo conditions, was therefore tested only on samples with the lowest concentration $(0.036 \%)$ of HaPANI and 
HaPPy (Figure 10). According to the results, it is obvious that cells were able to efficiently ingrow into the scaffold structure. This test showed not only that the scaffolds prepared here were cytocompatible with respect to basic parameters such as absence of cytotoxicity, but also that their bulk architecture created a friendly environment for cell ingrowth; thus, such scaffolds can be suitable for use in tissue engineering. Furthermore, their inherent conductivity, as a cell-instructive property, assured by the presence of CP-based colloids, opens up their potential use in a wide range of applications relating to the tissue engineering of electro-sensitive tissues.

\section{Conclusion}

The preparation and composition of scaffolds have been widely studied for decades, as they are critical factors conditioning the application of scaffolds in tissue engineering. In addition, scaffolds should not only be passive supporting materials for cells, but also enable preferable responses to external stimuli leading to cell-instructiveness. One of the cell-instructive cues of materials is electrical conductivity, which is especially advantageous when electro-sensitive tissues, such as heart, neuronal, or muscle tissues, are considered. The incorporation of conducting polymers into the structures of scaffolds is an advantageous way of inducing conductivity in the materials. The poor solubility of CPs in an aqueous environment and their limited miscibility with other hydrophilic polymer matrices makes the fabrication of scaffolds based solely on CPs difficult. The novelty of the here-presented fabrication techniques lies in their removal of these shortcomings by preparing colloidal particles based on the conducting polymers PANI and PPy and incorporating them into the structure of the biocompatible and biodegradable polymer sodium hyaluronate. In our study, two crosslinking methods were employed - namely, chemical and physical - and the influence of composition and the route of crosslinking on the final material, and the biological properties of the scaffolds were determined. In agreement with the presented data, it can be concluded that both used crosslinking techniques allow the efficient incorporation of colloidal particles into the bulk polymer, making thus the final composites conductive. The battery of characterization methods employed here revealed that the porosity, pore size, mechanical properties, and swelling of the studied samples were rather similar. Also, the results of cytotoxicity tests confirmed that the scaffolds were similarly cytocompatible with respect to this important characteristic. The effect of the lyophilization process on cytotoxicity was found to be greater than the crosslinking route used for preparation. Most crucially, tests conducted in bioreactors mimicking in vivo growth conditions showed that the scaffolds allow the adhesion and growth of cells on their surfaces; moreover, the cells were even able to ingrow into the structure of the scaffolds. The materials presented here thus represent electroconductive and cytocompatible composites with potentially cell-instructive properties.

\section{Declarations}

\section{Acknowledgement}


This work was supported by the Czech Science Foundation (20-28732S). This work was supported by the Ministry of Education, Youth and Sports of the Czech Republic - DKRVO (RP/CPS/2020/001)

\section{References}

1. Zhao, Y. et al. Application of conductive PPy/SF composite scaffold and electrical stimulation for neural tissue engineering., 255, 120164 (2020).

2. Elzinga, K. et al. Brief electrical stimulation improves nerve regeneration after delayed repair in Sprague Dawley rats. Exp. Neurol, 269, 142-153 (2015).

3. Deng, Z., Guo, Y., Zhao, X., Ma, P. X. \& Guo, B. Multifunctional Stimuli-Responsive Hydrogels with SelfHealing, High Conductivity, and Rapid Recovery through Host-Guest Interactions. Chem. Mater, 30, 1729-1742 (2018).

4. Saberi, A., Jabbari, F., Zarrintaj, P., Saeb, M. R. \& Mozafari, M. Electrically Conductive Materials: Opportunities and Challenges in Tissue Engineering. Biomolecules vol. 9 (2019)

5. Paulsen, B. D., Tybrandt, K., Stavrinidou, E. \& Rivnay, J. Organic mixed ionic-electronic conductors. Nat. Mater, 19, 13-26 (2020).

6. Sikorski, P. Electroconductive scaffolds for tissue engineering applications. Biomater. Sci, 8, 55835588 (2020).

7. Cui, Z. et al. Polypyrrole-chitosan conductive biomaterial synchronizes cardiomyocyte contraction and improves myocardial electrical impulse propagation. Theranostics, 8, 2752-2764 (2018).

8. Skopalová, K. et al. Modulation of Differentiation of Embryonic Stem Cells by Polypyrrole: The Impact on Neurogenesis.International Journal of Molecular Sciencesvol. 22 (2021)

9. Humpolicek, P., Kasparkova, V., Saha, P. \& Stejskal, J. Biocompatibility of polyaniline. Synth. Met, 162, 722-727 (2012).

10. Kucekova, Z. et al. Colloidal polyaniline dispersions: Antibacterial activity, cytotoxicity and neutrophil oxidative burst. Colloids Surfaces B Biointerfaces, 116, 411-417 (2014).

11. Jasenská, D. et al. Conducting composite films based on chitosan or sodium hyaluronate. Properties and cytocompatibility with human induced pluripotent stem cells. Carbohydr. Polym.253, (2021)

12. Tavsanli, B. \& Okay, O. Mechanically strong hyaluronic acid hydrogels with an interpenetrating network structure. Eur. Polym. J, 94, 185-195 (2017).

13. Björninen, M. et al. Comparison of Chondroitin Sulfate and Hyaluronic Acid Doped Conductive Polypyrrole Films for Adipose Stem Cells. Ann. Biomed. Eng, 42, 1889-1900 (2014).

14. Hemshekhar, M. et al. Emerging roles of hyaluronic acid bioscaffolds in tissue engineering and regenerative medicine. Int. J. Biol. Macromol, 86, 917-928 (2016).

15. Collins, M. N. \& Birkinshaw, C. Hyaluronic acid based scaffolds for tissue engineering-A review. Carbohydr. Polym, 92, 1262-1279 (2013).

16. Hu, X. et al. Biodegradable poly (lactic acid-co-trimethylene carbonate)/chitosan microsphere scaffold with shape-memory effect for bone tissue engineering. Colloids Surfaces B Biointerfaces, 
195,111218 (2020).

17. Collier, J. H., Camp, J. P., Hudson, T. W. \& Schmidt, C. E. Synthesis and characterization of polypyrrole-hyaluronic acid composite biomaterials for tissue engineering applications. J. Biomed. Mater. Res, 50, 574-584 (2000).

18. Snetkov, P., Zakharova, K., Morozkina, S., Olekhnovich, R. \& Uspenskaya, M. Hyaluronic Acid: The Influence of Molecular Weight on Structural, Physical, Physico-Chemical, and Degradable Properties of Biopolymer. Polymers vol. 12 (2020)

19. Kim, S. et al. Versatile biomimetic conductive polypyrrole films doped with hyaluronic acid of different molecular weights. Acta Biomater, 80, 258-268 (2018).

20. Shin, J. et al. Three-Dimensional Electroconductive Hyaluronic Acid Hydrogels Incorporated with Carbon Nanotubes and Polypyrrole by Catechol-Mediated Dispersion Enhance Neurogenesis of Human Neural Stem Cells., 18, 3060-3072 (2017).

21. Texidó, R., Orgaz, A., Ramos-Pérez, V. \& Borrós, S. Stretchable conductive polypyrrole films modified with dopaminated hyaluronic acid. Mater. Sci. Eng. C, 76, 295-300 (2017).

22. Alves, T. et al. Biomimetic dense lamellar scaffold based on a colloidal complex of the polyaniline (PANi) and biopolymers for electroactive and physiomechanical stimulation of the myocardial. Colloids Surfaces A Physicochem. Eng. Asp, 579, 123650 (2019).

23. Gřundělová, L. et al. Viscoelastic and mechanical properties of hyaluronan films and hydrogels modified by carbodiimide. Carbohydr. Polym, 119, 142-148 (2015).

24. Holloway, J. L., Lowman, A. M. \& Palmese, G. R. The role of crystallization and phase separation in the formation of physically cross-linked PVA hydrogels., 9, 826-833 (2013).

25. Zhang, F., Wu, J., Kang, D. \& Zhang, H. Development of a complex hydrogel of hyaluronan and PVA embedded with silver nanoparticles and its facile studies on Escherichia coli. J. Biomater. Sci. Polym. $E d, 24,1410-1425$ (2013).

26. Oh, S. H., An, D. B., Kim, T. H. \& Lee, J. H. Wide-range stiffness gradient PVA/HA hydrogel to investigate stem cell differentiation behavior. Acta Biomater, 35, 23-31 (2016).

27. Kašpárková, V. et al. Polyaniline colloids stabilized with bioactive polysaccharides: Non-cytotoxic antibacterial materials. Carbohydr. Polym, 219, 423-430 (2019).

28. Kašpárková, V. et al. Exploring the Critical Factors Limiting Polyaniline Biocompatibility. Polymers vol. 11(2019)

29. Lewandowska, K. Miscibility Studies of Hyaluronic Acid and Poly(Vinyl Alcohol) Blends in Various Solvents.Materialsvol.13(2020)

30. Hassan, C. M. \& Peppas, N. a. Biopolymers · PVA Hydrogels, Anionic Polymerisation Nanocomposites.Biopolymers · PVA Hydrogels, Anionic Polymerisation Nanocompositesvol. 153 (2000)

31. Humpolíček, P. et al. The biocompatibility of polyaniline and polypyrrole: A comparative study of their cytotoxicity, embryotoxicity and impurity profile. Mater. Sci. Eng. C, 91, 303-310 (2018). 
Figures
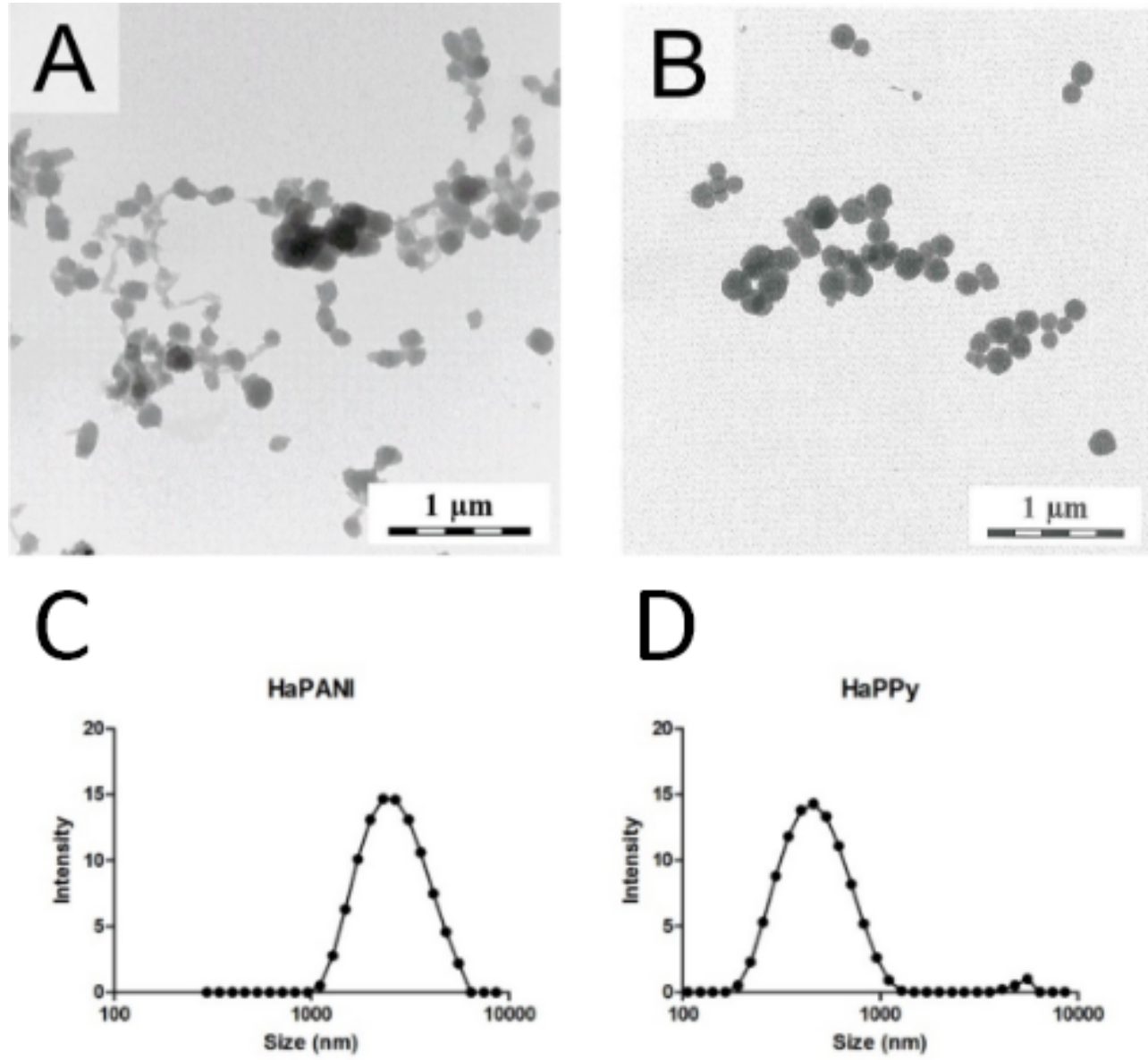

Figure 1

Transmission electron micrographs of CP-based colloidal particles stabilized with sodium hyaluronate HaPANI (A), HaPPy (B); and particle size distribution curves XX of HaPANI (C), HaPPy (D). 


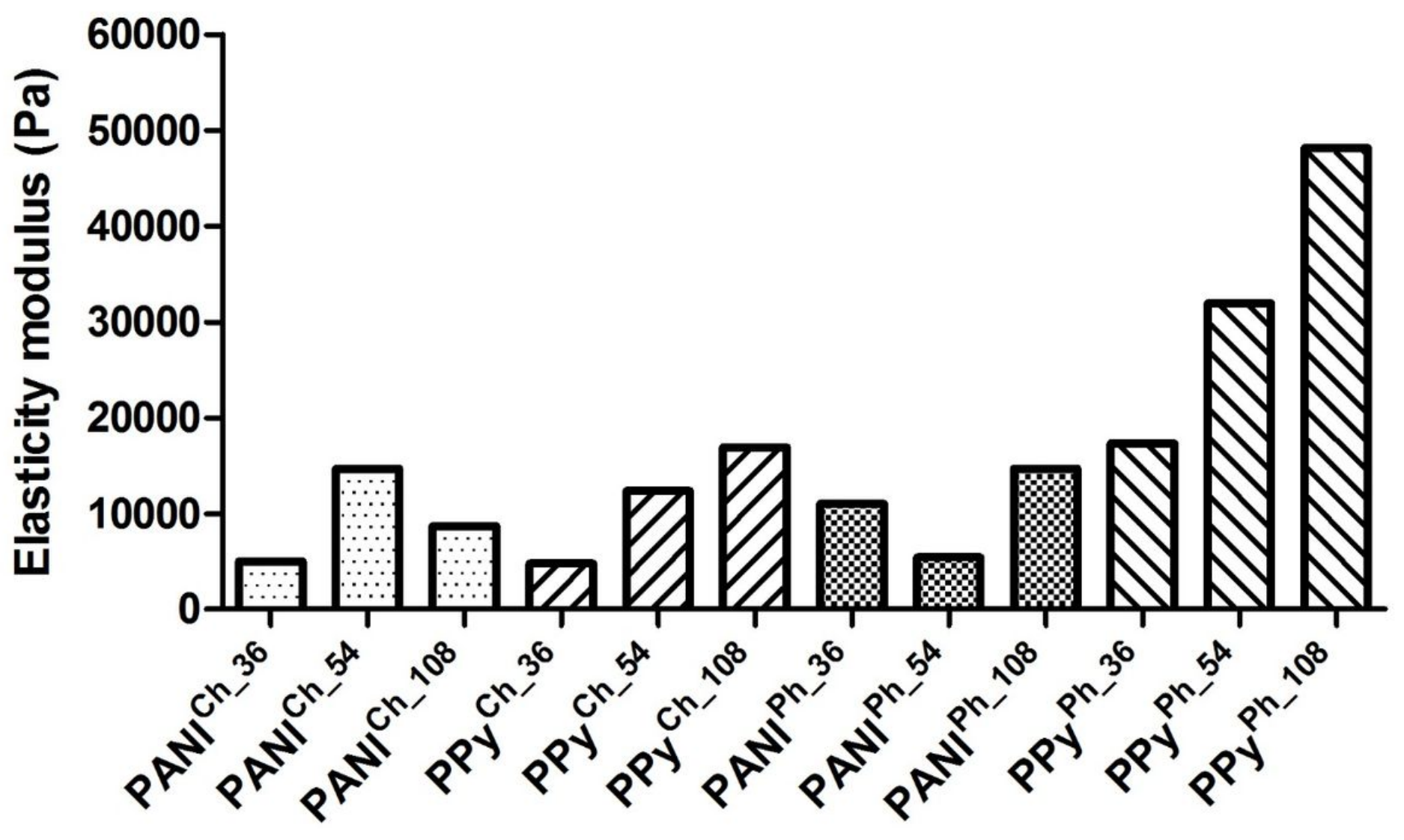

Figure 2

Elasticity moduli calculated for physically $(\mathrm{PH})$ and chemically $(\mathrm{CH})$ crosslinked scaffolds 


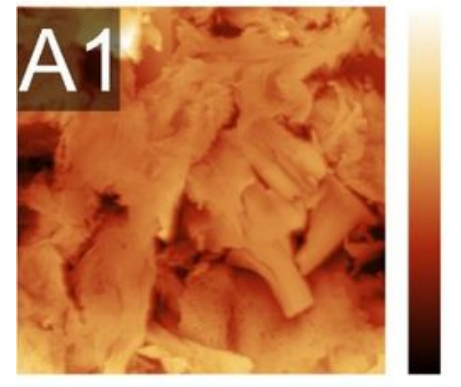

$\mathrm{Sa}=1.2 \mathrm{~mm} \quad 0 \mu \mathrm{m}$

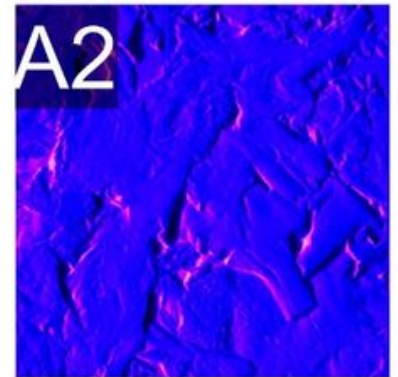

$A v=2.1 \mathrm{pA}$

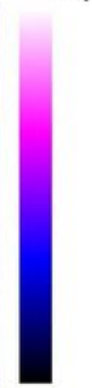

$0 \mathrm{pA}$

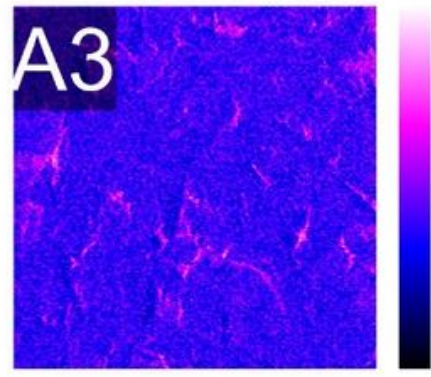

$A v .=5.4 \mathrm{pA} \quad 0 \mathrm{pA}$

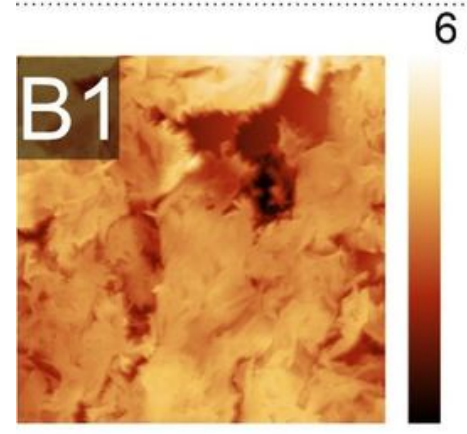

$6 \mu \mathrm{m}$

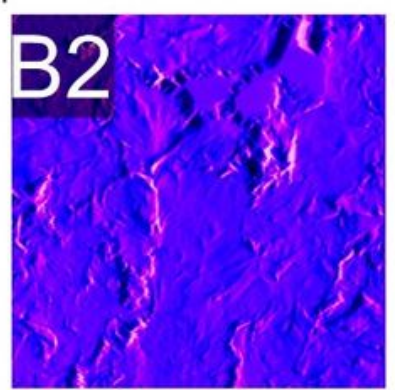

$\mathrm{Sa}=0.6 \mathrm{~mm} \quad 0 \mu \mathrm{m}$

$6 \mu \mathrm{m}$
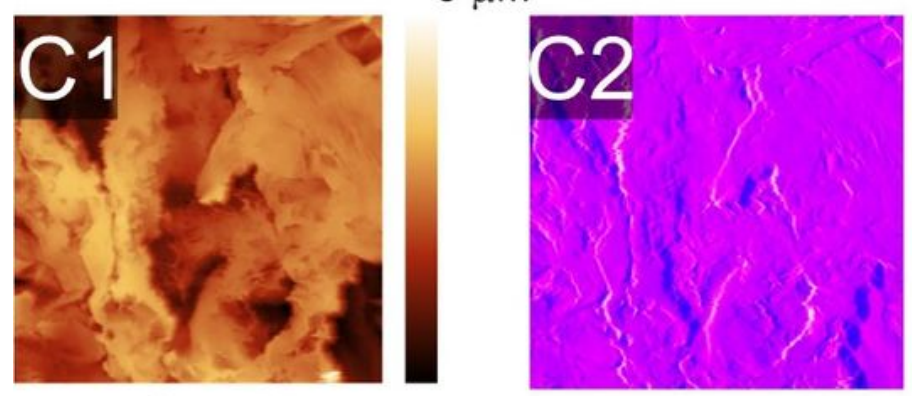

$\mathrm{Sa}=0.7 \mathrm{~mm} \quad 0 \mu \mathrm{m}$

$$
\text { Av. }=2.9 \mathrm{pA} \quad 0 \mathrm{pA}
$$

$8 \mathrm{pA}$
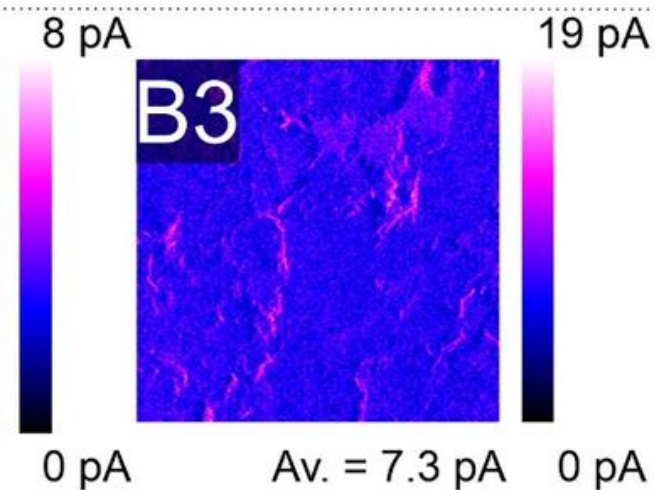

$4 \mathrm{pA}$

$3 \mathrm{pA}$

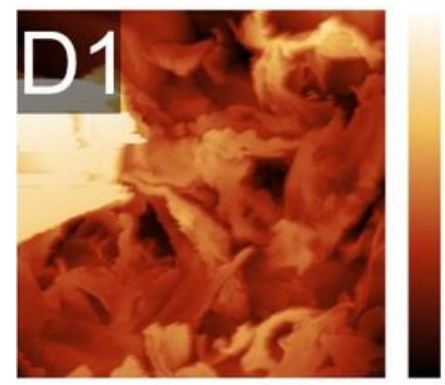

$8 \mu \mathrm{m}$
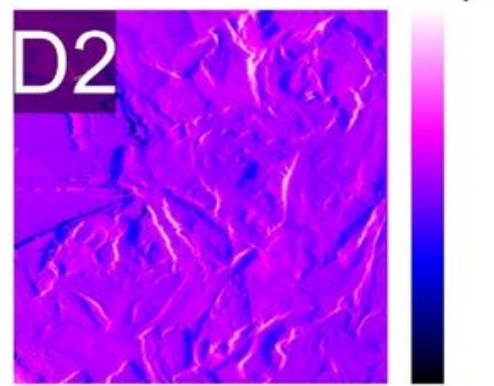

$\mathrm{Sa}=1.2 \mathrm{~mm} \quad 0 \mu \mathrm{m}$

$A v .=2.1 \mathrm{pA} \quad 0 \mathrm{pA}$

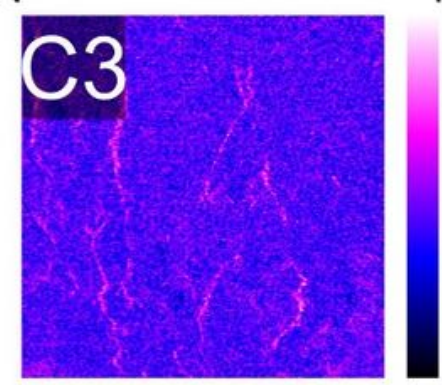

$12 \mathrm{pA}$

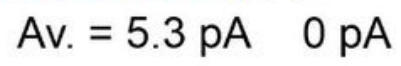

$11 \mathrm{pA}$
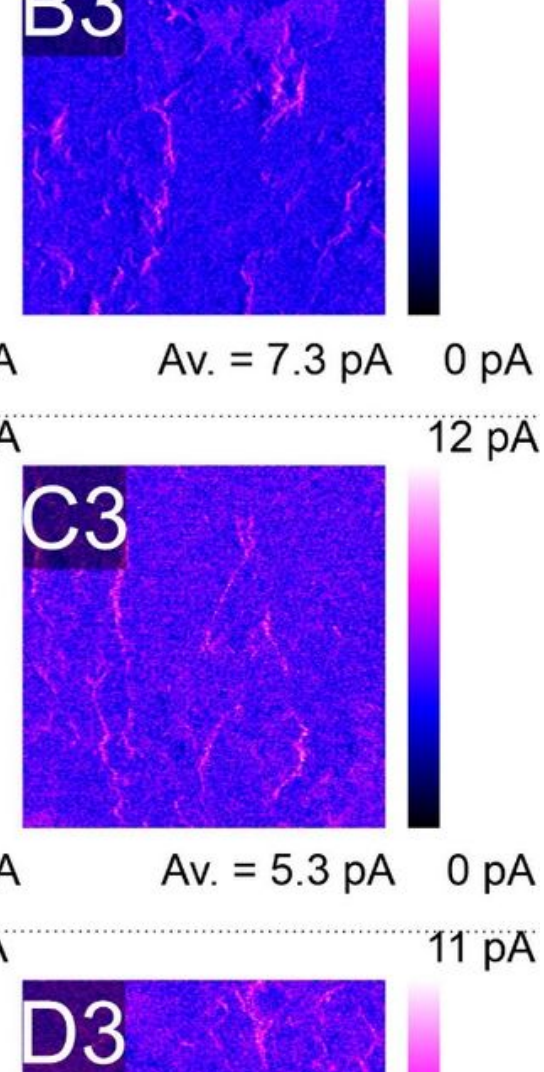$$
\text { Av. }=5.3 \mathrm{pA} \quad 0 \mathrm{pA}
$$

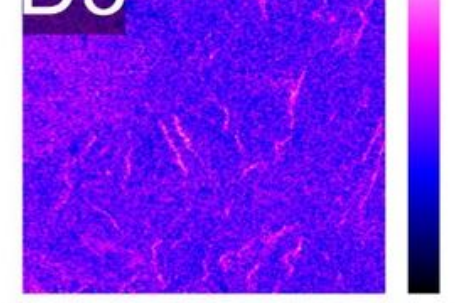

$$
\text { Av. }=5.4 \mathrm{pA} \quad 0 \mathrm{pA}
$$

Figure 3

AFM images of HaPANICh (A), HaPPyCh (B), HaPANIPh (C), HaPPyPh (D). The topography (1), TUNA current (2), and peak current (3) are presented for every sample 

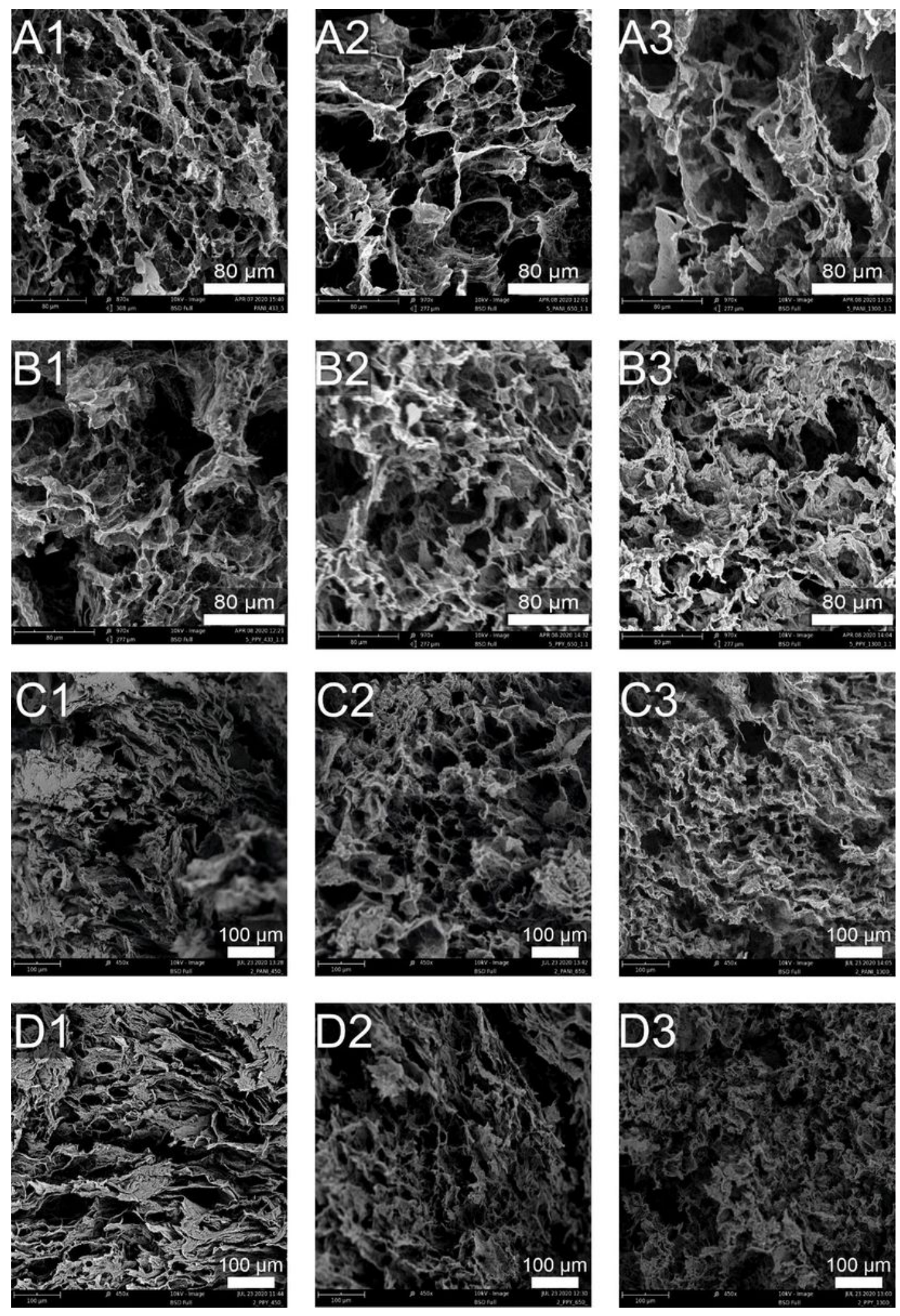

Figure 4

SEM photomicrographs of lyophilized HaPANICh (A), HaPPyCh (B), HaPANIPh (C) and HaPPyPh (C) containing colloid concentrations of 0.036 (1), 0.054 (2), or $0,108(3) \%$. 


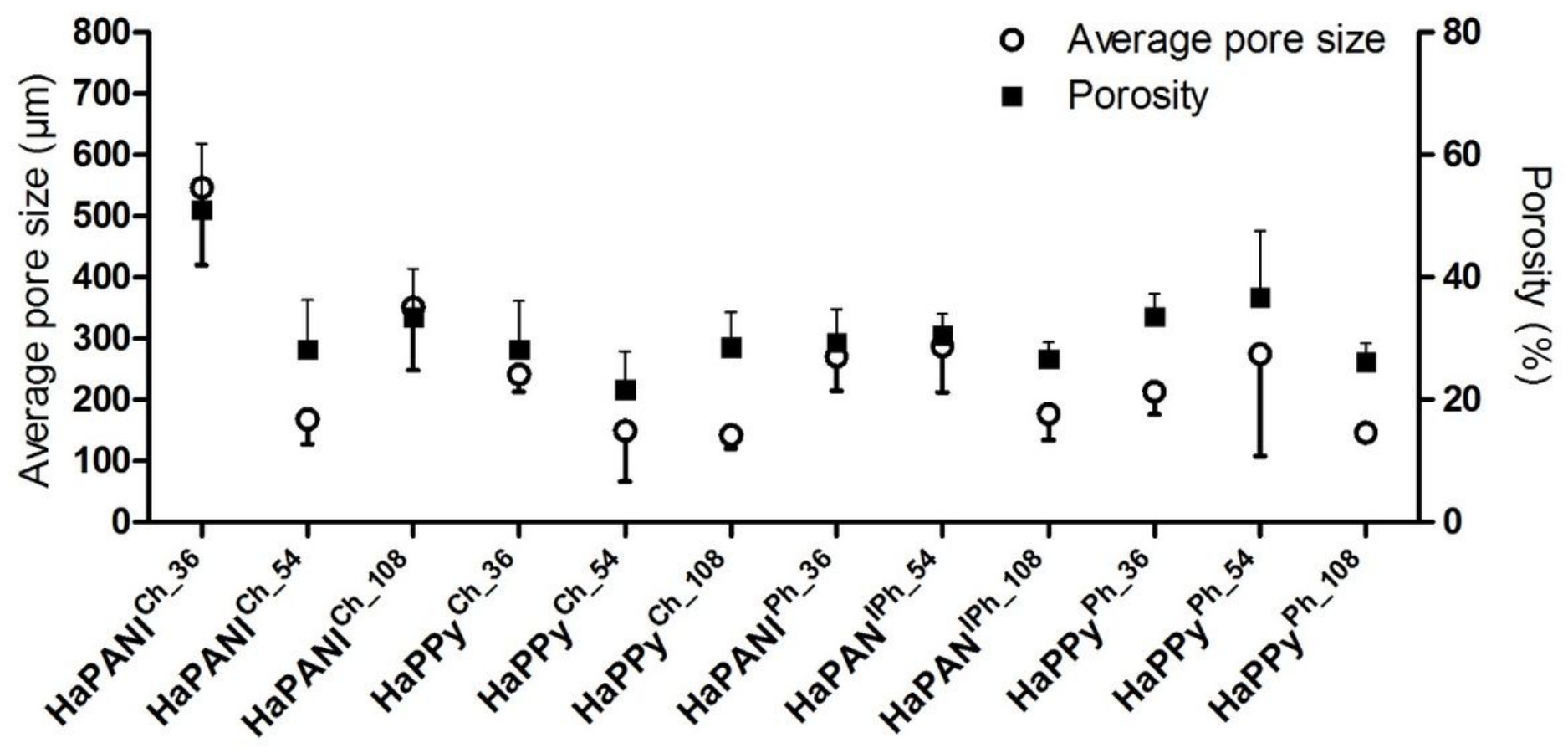

Figure 5

Average pore size and average porosity of the scaffolds as determined by image analysis according to ASTM E-112. 

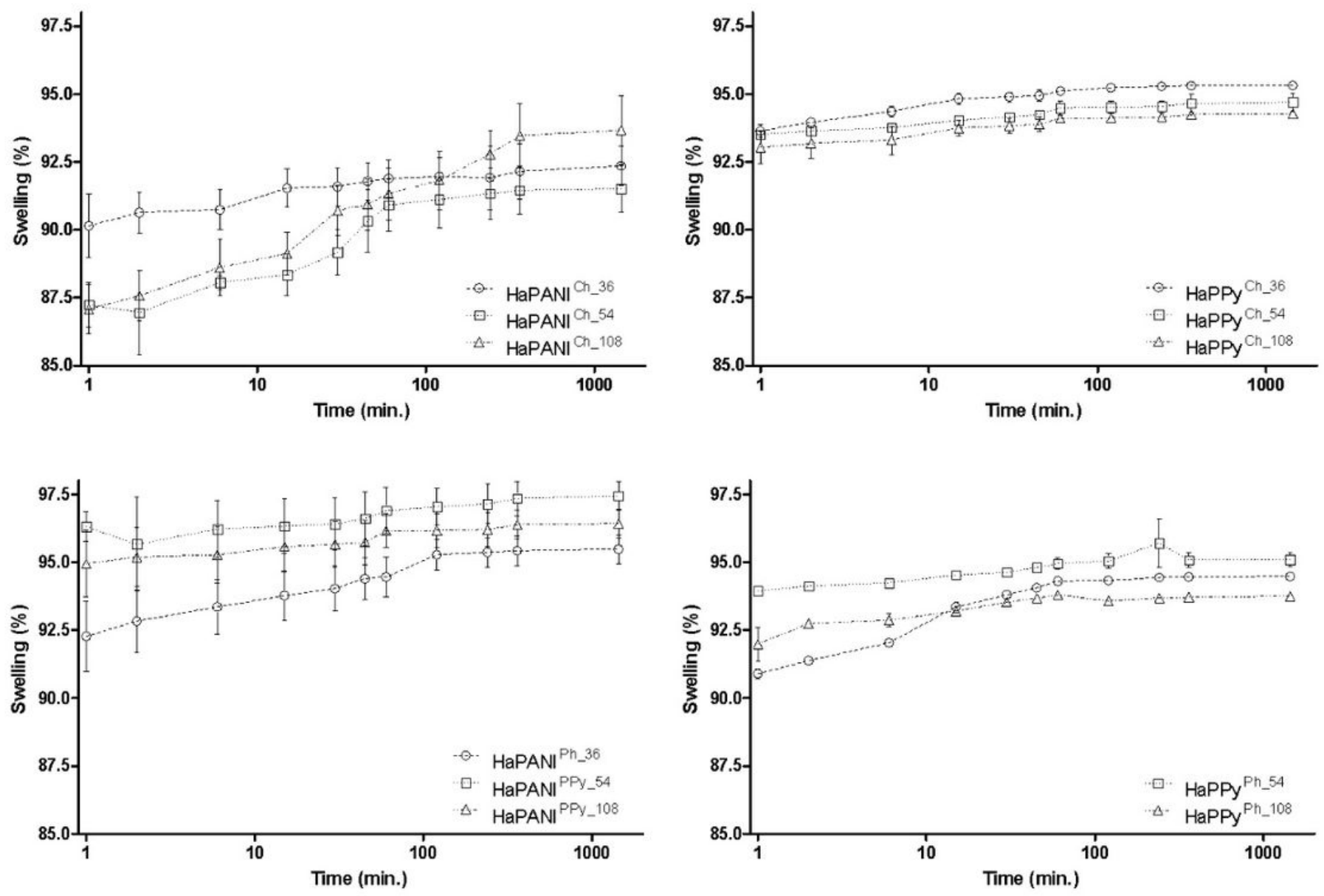

\section{Figure 6}

Swelling behaviour, expressed as average swelling degree. 


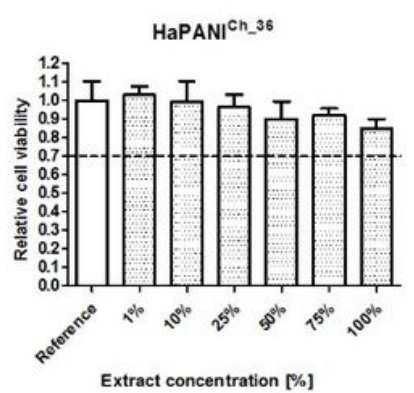

HaPANI ${ }^{\text {ch_s }}{ }^{4}$

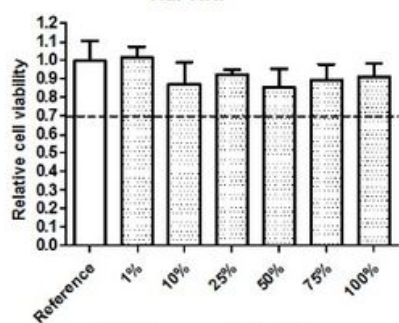

Extract concentration $[\%]$

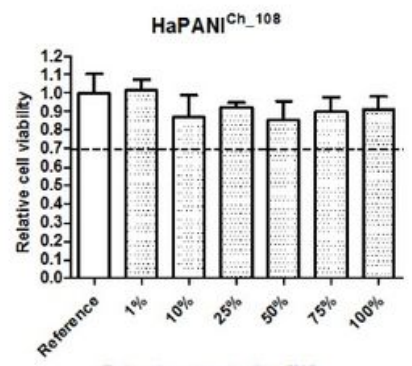

Extract concentration [\%]

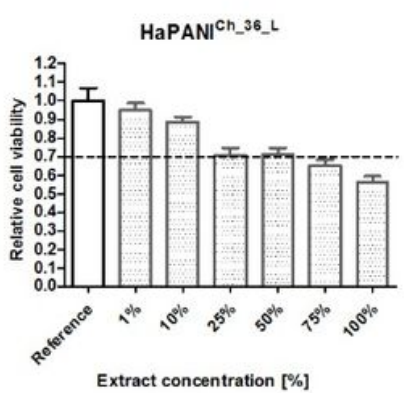

HaPANI ${ }^{\text {Ch_54_L }}$
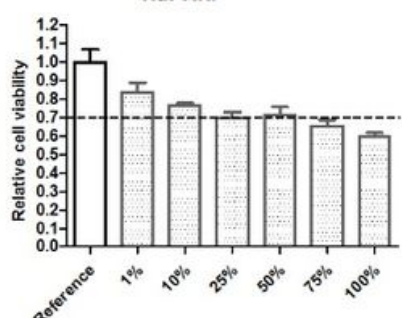

Extract concentration $[\%]$

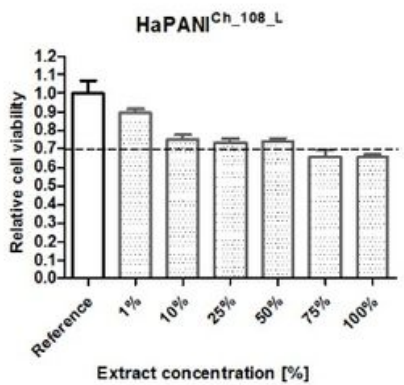

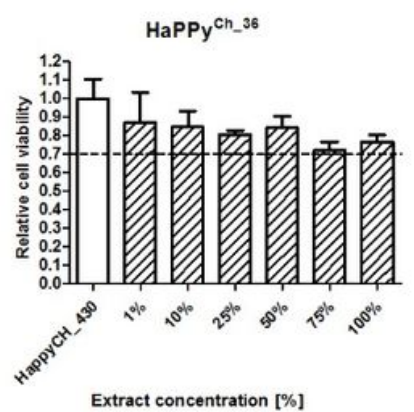

HaPPy ${ }^{\text {ch_54 }}$

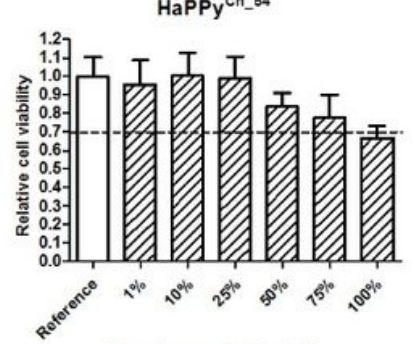

Extract concentration [\%]

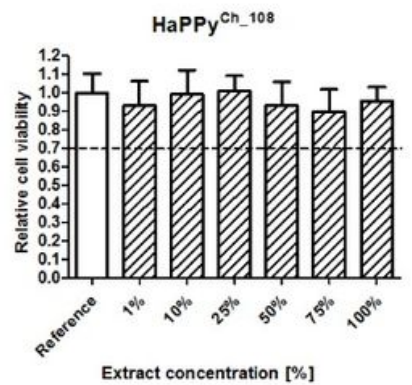

HaPPy ${ }^{\text {Ch_36_L }}$

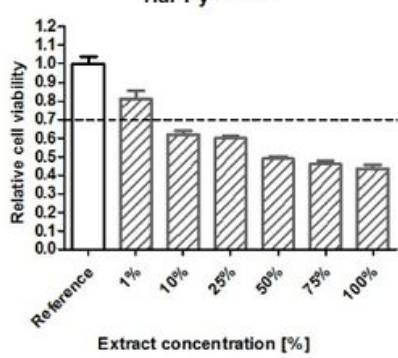

HaPPy ch-64_L

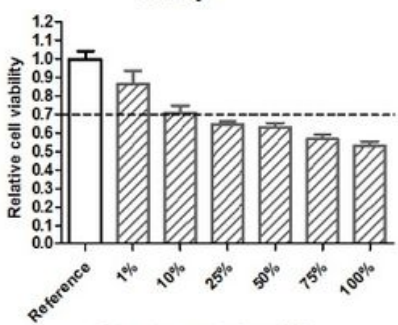

Extract concentration [\%]

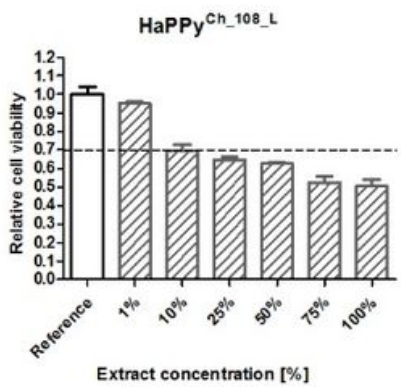

Figure 7

The cytotoxicity of chemically crosslinked scaffolds. The dashed line shows the threshold of cytotoxicity (70\% viability of cells). 

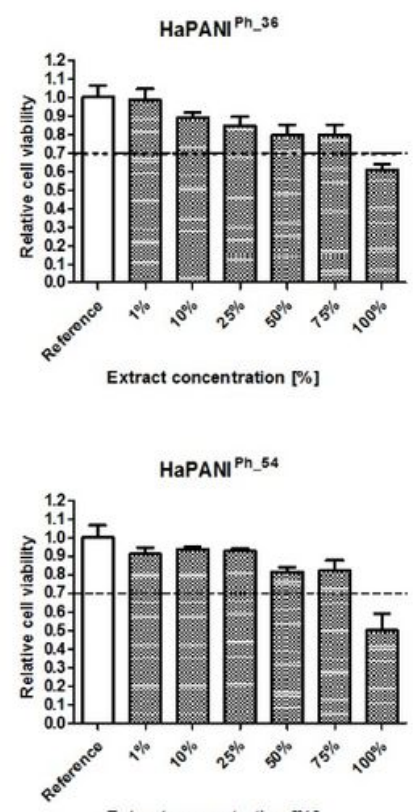

Extract concentration [\%]

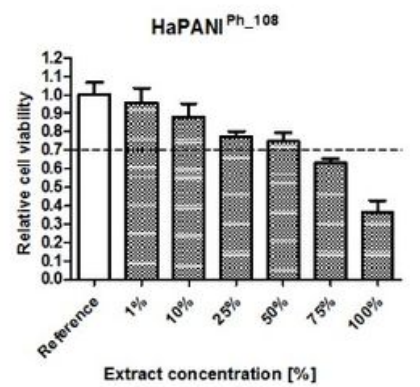

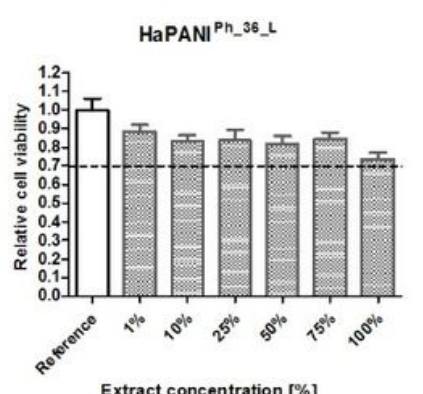

Extract concentration $[\%]$

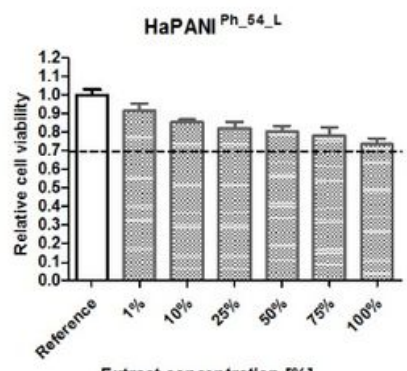

Extract concentration $[\%]$

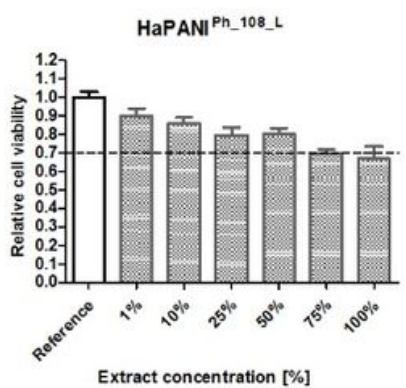

HaPPy $^{\text {Ph_36 }}$

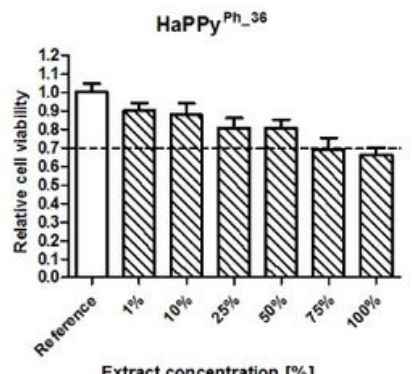

HaPPy ${ }^{\text {Ph_54 }}$

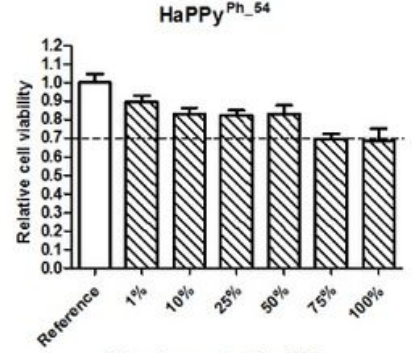

Extract concentration [\%]

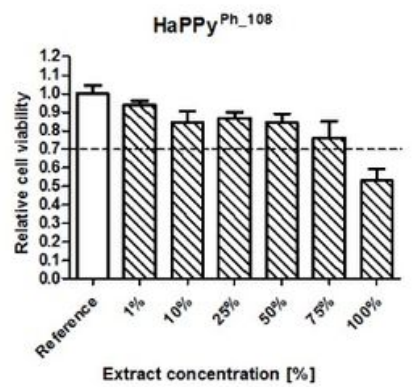

HaPPy ${ }^{\text {Ph_36_L }}$

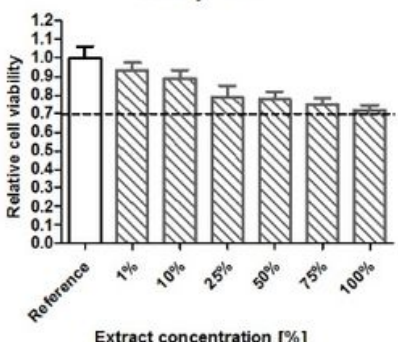

HaPPy Ph_54_L

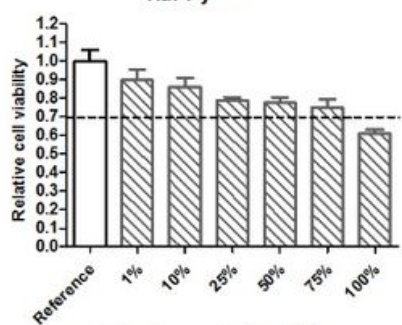

Extract concentration [\%]

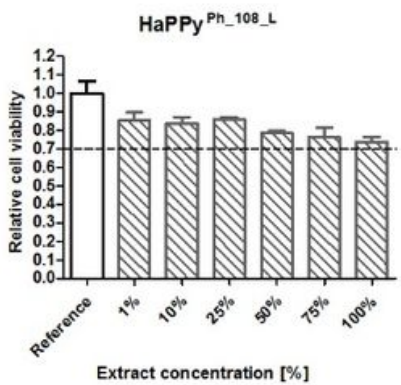

Figure 8

The cytotoxicity of physically crosslinked scaffolds. The dashed line shows the threshold of cytotoxicity (70\% viability of cells compare to reference). 

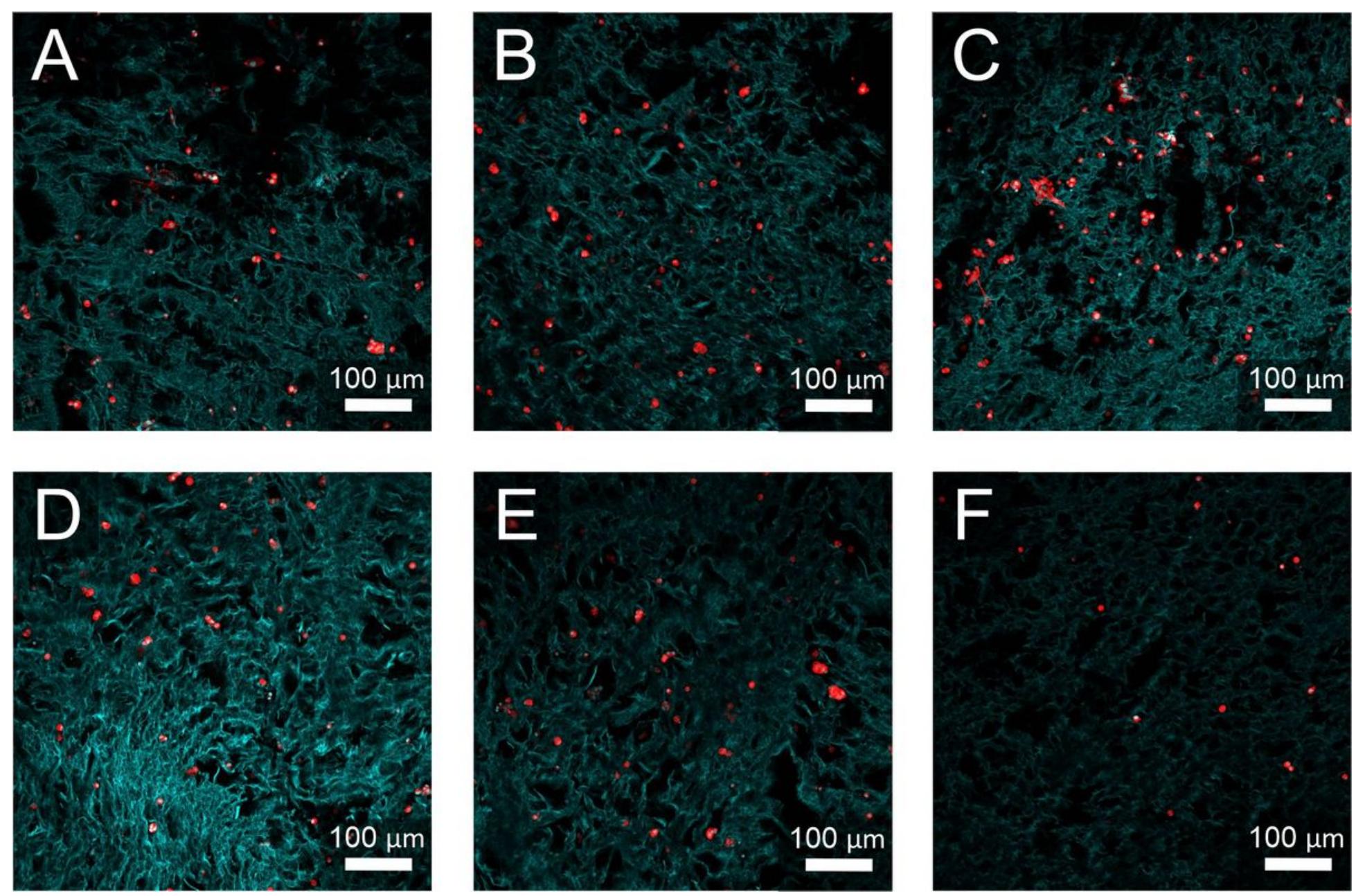

Figure 9

The adhesion and growth of cells on the surface of lyophilised HaPANICh_36_L (A); HaPANICh_54_L (B); HaPANICh_108_L (C); HaPPyCh_36_L (D), HaPPy Ch_54_L (E), HaPPy Ch_108_L (F). Cell nuclei were counterstained with Hoechst (blue); the cytoskeleton was counterstained with ActinRed (red). The green mass represents the hydrogel structure. 

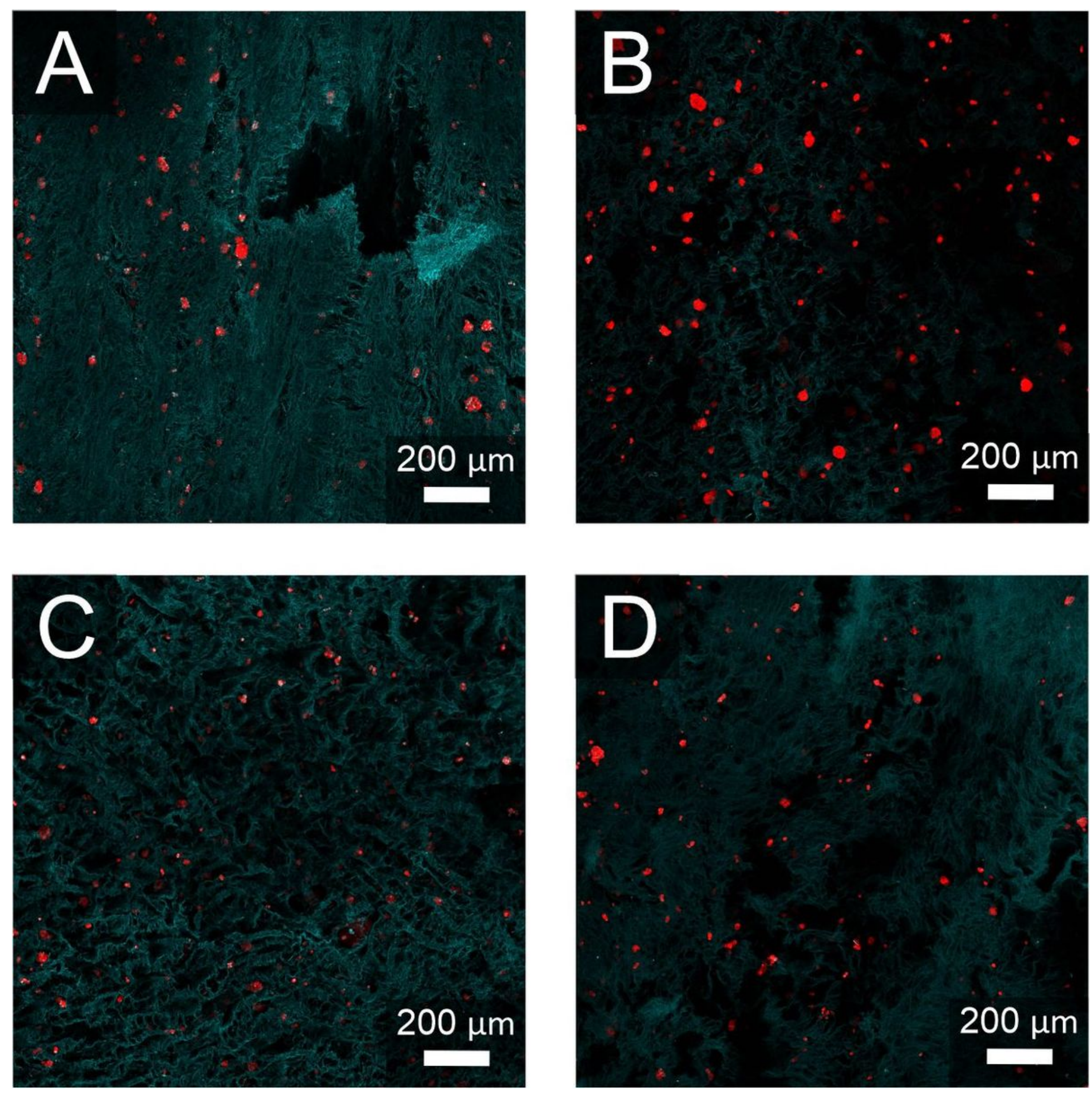

Figure 10

The cell ingrowth into the structure of HaPANI Ph_36_L (A); HaPPy Ph_36_L (B); HaPANI Ch_36_L (C); HaPPy Ch_36_L (D) Cell nuclei were counterstained with Hoechst (blue); the cytoskeleton was counterstained with actin red (red). The green mass represents the hydrogel structure. 